\title{
The Internet of Things
}

How the Next Evolution of the Internet Is Changing Everything

Author

Dave Evans

April 2011 


\section{The Internet of Things}

\section{How the Next Evolution of the Internet Is Changing Everything}

The Internet of Things (IoT), sometimes referred to as the Internet of Objects, will change everything -including ourselves. This may seem like a bold statement, but consider the impact the Internet already has had on education, communication, business, science, government, and humanity. Clearly, the Internet is one of the most important and powerful creations in all of human history.

Now consider that loT represents the next evolution of the Internet, taking a huge leap in its ability to gather, analyze, and distribute data that we can turn into information, knowledge, and, ultimately, wisdom. In this context, loT becomes immensely important.

Already, loT projects are under way that promise to close the gap between poor and rich, improve distribution of the world's resources to those who need them most, and help us understand our planet so we can be more proactive and less reactive. Even so, several barriers exist that threaten to slow loT development, including the transition to IPv6, having a common set of standards, and developing energy sources for millions-even billions-of minute sensors.

However, as businesses, governments, standards bodies, and academia work together to solve these challenges, loT will continue to progress. The goal of this paper, therefore, is to educate you in plain and simple terms so you can be well versed in loT and understand its potential to change everything we know to be true today.

\section{loT Today}

As with many new concepts, loT's roots can be traced back to the Massachusetts Institute of Technology (MIT), from work at the Auto-ID Center. Founded in 1999, this group was working in the field of networked radio frequency identification (RFID) and emerging sensing technologies. The labs consisted of seven research universities located across four continents. These institutions were chosen by the Auto-ID Center to design the architecture for loT.

Before we talk about the current state of loT, it is important to agree on a definition. According to the Cisco Internet Business Solutions Group (IBSG), IOT is simply the point in time when more "things or objects" were connected to the Internet than people. ${ }^{2}$

In 2003, there were approximately 6.3 billion people living on the planet and 500 million devices connected to the Internet. ${ }^{3}$ By dividing the number of connected devices by the world population, we find that there was less than one (0.08) device for every person. Based on Cisco IBSG's definition, IoT didn't yet exist in 2003 because the number of connected things was relatively small given that ubiquitous devices such as smartphones were just being introduced. For example, Steve Jobs, Apple's CEO, didn't unveil the iPhone until January 9, 2007 at the Macworld conference. ${ }^{4}$ 
Explosive growth of smartphones and tablet PCs brought the number of devices connected to the Internet to 12.5 billion in 2010, while the world's human population increased to 6.8 billion, making the number of connected devices per person more than 1 (1.84 to be exact) for the first time in history. ${ }^{5}$

\begin{abstract}
Methodology
In January 2009, a team of researchers in China studied Internet routing data in six-month intervals, from December 2001 to December 2006. Similar to the properties of Moore's Law, their findings showed that the Internet doubles in size every 5.32 years. Using this figure in combination with the number of devices connected to the Internet in 2003 (500 million, as determined by Forrester Research), and the world population according to the U.S. Census Bureau, Cisco IBSG estimated the number of connected devices per person. ${ }^{6}$
\end{abstract}

Refining these numbers further, Cisco IBSG estimates loT was "born" sometime between 2008 and 2009 (see Figure 1). Today, loT is well under way, as initiatives such as Cisco's Planetary Skin, smart grid, and intelligent vehicles continue to progress.?

Figure 1. The Internet of Things Was “Born” Between 2008 and 2009

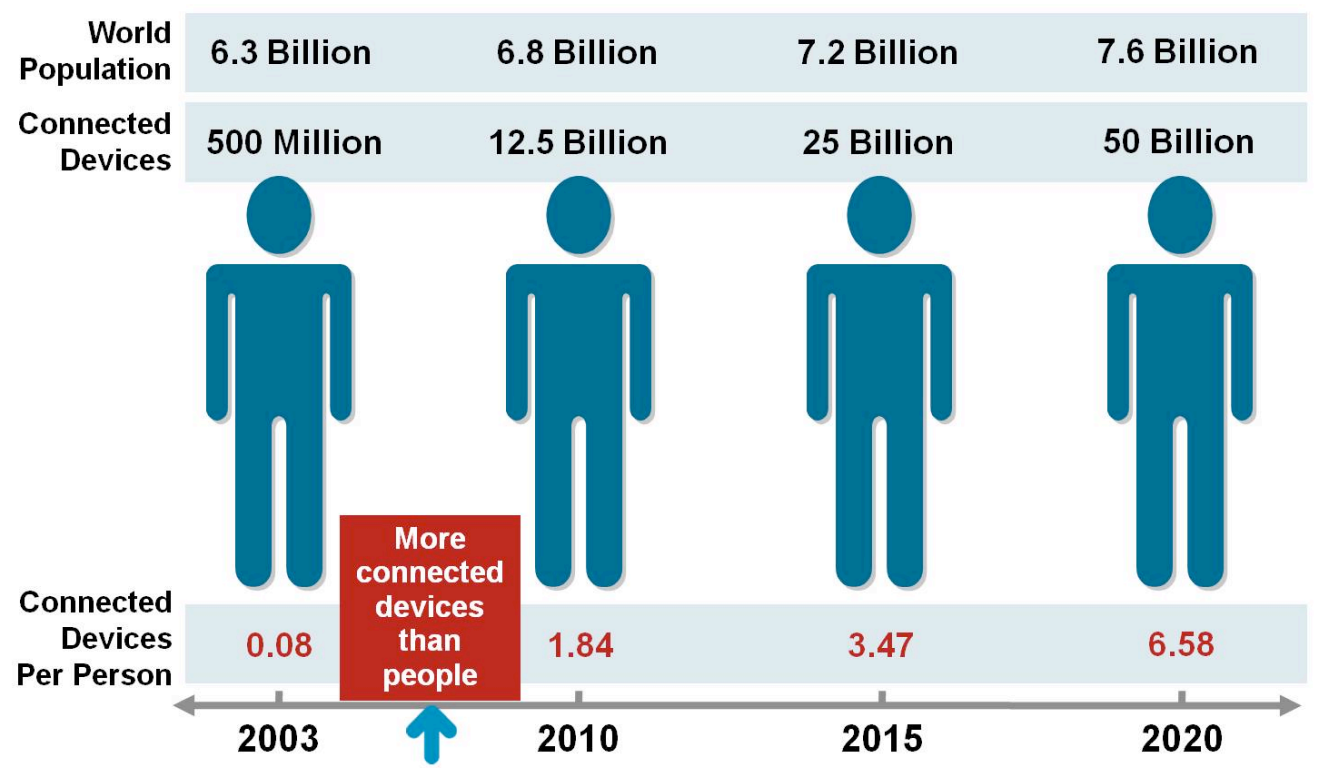

Source: Cisco IBSG, April 2011

Looking to the future, Cisco IBSG predicts there will be 25 billion devices connected to the Internet by 2015 and 50 billion by 2020. It is important to note that these estimates do not take into account rapid advances in Internet or device technology; the numbers presented are based on what is known to be true today.

Additionally, the number of connected devices per person may seem low. This is because the calculation is based on the entire world population, much of which is not yet connected to the Internet. By reducing the population sample to people actually connected to the Internet, the number of connected devices per person rises dramatically. For example, we know that 
approximately 2 billion people use the Internet today. ${ }^{8}$ Using this figure, the number of connected devices per person jumps to 6.25 in 2010, instead of 1.84 .

Of course, we know nothing remains static, especially when it comes to the Internet. Initiatives and advances, such as Cisco's Planetary Skin, HP's central nervous system for the earth (CeNSE), and smart dust, have the potential to add millions - even billions-of sensors to the Internet. ${ }^{9}$ As cows, water pipes, people, and even shoes, trees, and animals become connected to loT, the world has the potential to become a better place.

"With a trillion sensors embedded in the environment-all connected by computing systems, software, and services - it will be possible to hear the heartbeat of the Earth, impacting human interaction with the globe as profoundly as the Internet has revolutionized communication."

Peter Hartwell

Senior Researcher, HP Labs

\section{IoT as a Network of Networks}

Currently, loT is made up of a loose collection of disparate, purpose-built networks. Today's cars, for example, have multiple networks to control engine function, safety features, communications systems, and so on. Commercial and residential buildings also have various control systems for heating, venting, and air conditioning (HVAC); telephone service; security; and lighting. As loT evolves, these networks, and many others, will be connected with added security, analytics, and management capabilities (see Figure 2). This will allow loT to become even more powerful in what it can help people achieve.

Figure 2. IoT Can Be Viewed as a Network of Networks

\section{Internet of Things}

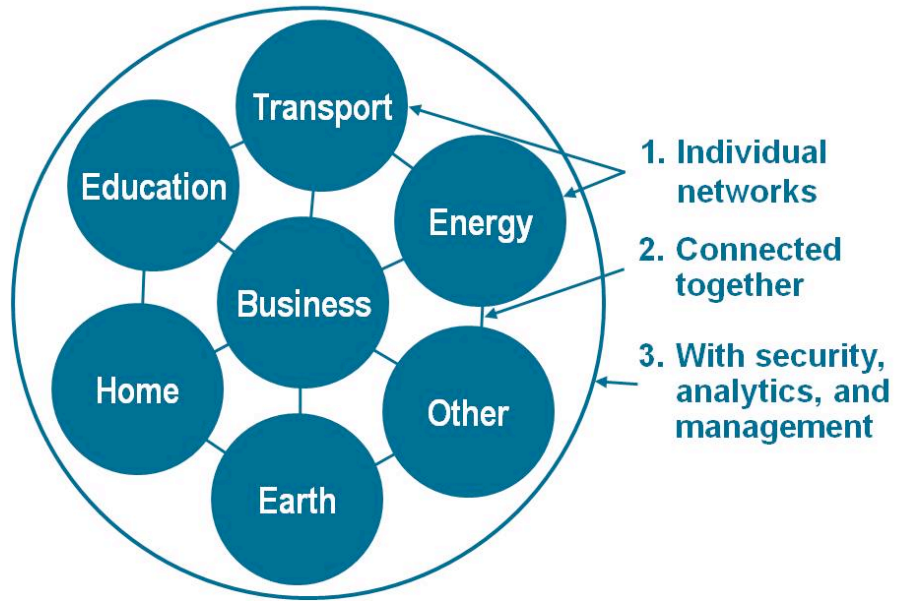

Source: Cisco IBSG, April 2011 
Interestingly, this situation mirrors what the technology industry experienced in the early days of networking. In the late 1980s and early 1990s, Cisco, for example, established itself by bringing disparate networks together with multi-protocol routing, eventually leading to IP as the common networking standard. With loT, history is repeating itself, albeit on a much grander scale.

\section{Why Is loT Important?}

Before we can begin to see the importance of loT, it is first necessary to understand the differences between the Internet and the World Wide Web (or web) —terms that are often used interchangeably. The Internet is the physical layer or network made up of switches, routers, and other equipment. Its primary function is to transport information from one point to another quickly, reliably, and securely. The web, on the other hand, is an application layer that operates on top of the Internet. Its primary role is to provide an interface that makes the information flowing across the Internet usable.

\section{Evolution of the Web Versus the Internet}

The web has gone through several distinct evolutionary stages:

Stage 1. First was the research phase, when the web was called the Advanced Research Projects Agency Network (ARPANET). During this time, the web was primarily used by academia for research purposes.

Stage 2. The second phase of the web can be coined "brochureware." Characterized by the domain name "gold rush," this stage focused on the need for almost every company to share information on the Internet so that people could learn about products and services.

Stage 3. The third evolution moved the web from static data to transactional information, where products and services could be bought and sold, and services could be delivered. During this phase, companies like eBay and Amazon.com exploded on the scene. This phase also will be infamously remembered as the "dot-com" boom and bust.

Stage 4. The fourth stage, where we are now, is the "social" or "experience" web, where companies like Facebook, Twitter, and Groupon have become immensely popular and profitable (a notable distinction from the third stage of the web) by allowing people to communicate, connect, and share information (text, photos, and video) about themselves with friends, family, and colleagues.

\section{loT: First Evolution of the Internet}

By comparison, the Internet has been on a steady path of development and improvement, but arguably hasn't changed much. It essentially does the same thing that it was designed to do during the ARPANET era. For example, in the early days, there were several communication protocols, including AppleTalk, Token Ring, and IP. Today, the Internet is largely standardized on IP.

In this context, loT becomes immensely important because it is the first real evolution of the Internet-a leap that will lead to revolutionary applications that have the potential to dramatically improve the way people live, learn, work, and entertain themselves. Already, loT has made the Internet sensory (temperature, pressure, vibration, light, moisture, stress), allowing us to become more proactive and less reactive. 
In addition, the Internet is expanding into places that until now have been unreachable. Patients are ingesting Internet devices into their own bodies to help doctors diagnose and determine the causes of certain diseases. ${ }^{10}$ Extremely small sensors can be placed on plants, animals, and geologic features, and connected to the Internet. ${ }^{11}$ At the other end of the spectrum, the Internet is going into space through Cisco's Internet Routing in Space (IRIS) program. $^{12}$

\section{We Evolve Because We Communicate}

Humans evolve because they communicate. Once fire was discovered and shared, for example, it didn't need to be rediscovered, only communicated. A more modern-day example is the discovery of the helix structure of DNA, molecules that carry genetic information from one generation to another. After the article was published in a scientific paper by James Watson and Francis Crick in April 1953, the disciplines of medicine and genetics were able to build on this information to take giant leaps forward. ${ }^{13}$

This principle of sharing information and building on discoveries can best be understood by examining how humans process data (see Figure 3). From bottom to top, the pyramid layers include data, information, knowledge, and wisdom. Data is the raw material that is processed into information. Individual data by itself is not very useful, but volumes of it can identify trends and patterns. This and other sources of information come together to form knowledge. In the simplest sense, knowledge is information of which someone is aware. Wisdom is then born from knowledge plus experience. While knowledge changes over time, wisdom is timeless, and it all begins with the acquisition of data.

Figure 3. Humans Turn Data into Wisdom

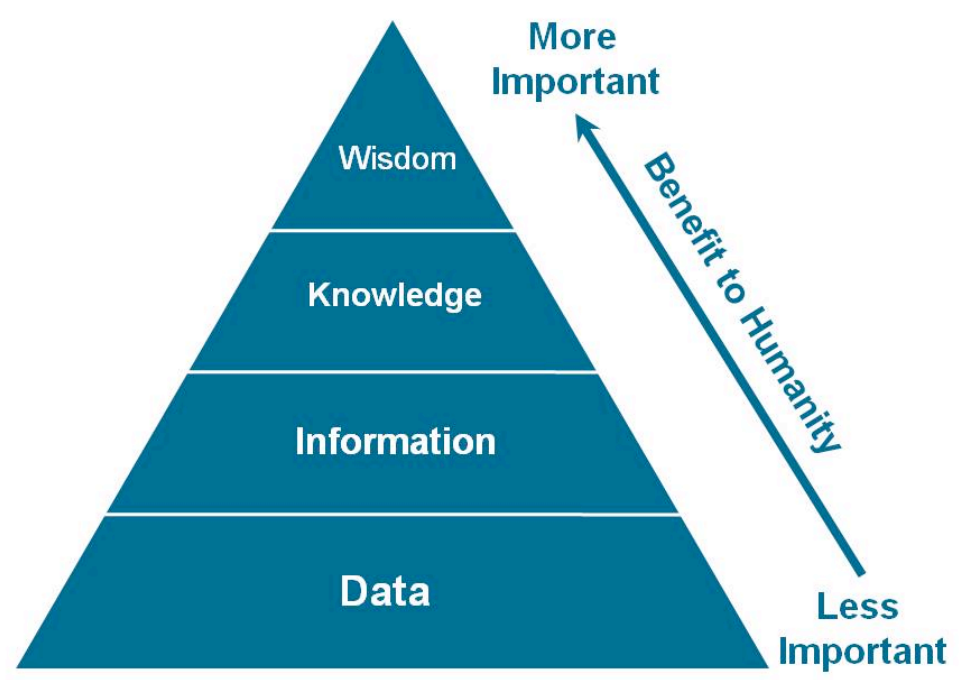

Source: Cisco IBSG, April 2011

It is also important to note there is a direct correlation between the input (data) and output (wisdom). The more data that is created, the more knowledge and wisdom people can obtain. loT dramatically increases the amount of data available for us to process. This, coupled with the Internet's ability to communicate this data, will enable people to advance even further. 


\section{IoT: Critical for Human Progression}

As the planet's population continues to increase, it becomes even more important for people to become stewards of the earth and its resources. In addition, people desire to live healthy, fulfilling, and comfortable lives for themselves, their families, and those they care about. By combining the ability of the next evolution of the Internet (IOT) to sense, collect, transmit, analyze, and distribute data on a massive scale with the way people process information, humanity will have the knowledge and wisdom it needs not only to survive, but to thrive in the coming months, years, decades, and centuries.

\section{IoT Applications: What Cows, Water Pipes, and People Have in Common}

When we crossed the threshold of connecting more objects than people to the Internet, a huge window of opportunity opened for the creation of applications in the areas of automation, sensing, and machine-to-machine communication. In fact, the possibilities are almost endless. The following examples highlight some of the ways loT is changing people's lives for the better.

\section{Holy Cow!}

In the world of loT, even cows will be connected. A special report in The Economist titled "Augmented Business" described how cows will be monitored (see Figure 4). Sparked, a Dutch start-up company, implants sensors in the ears of cattle. This allows farmers to monitor cows' health and track their movements, ensuring a healthier, more plentiful supply of meat for people to consume. On average, each cow generates about 200 megabytes of information a year. ${ }^{14}$

Figure 4. Even Cows Will Have Sensors.

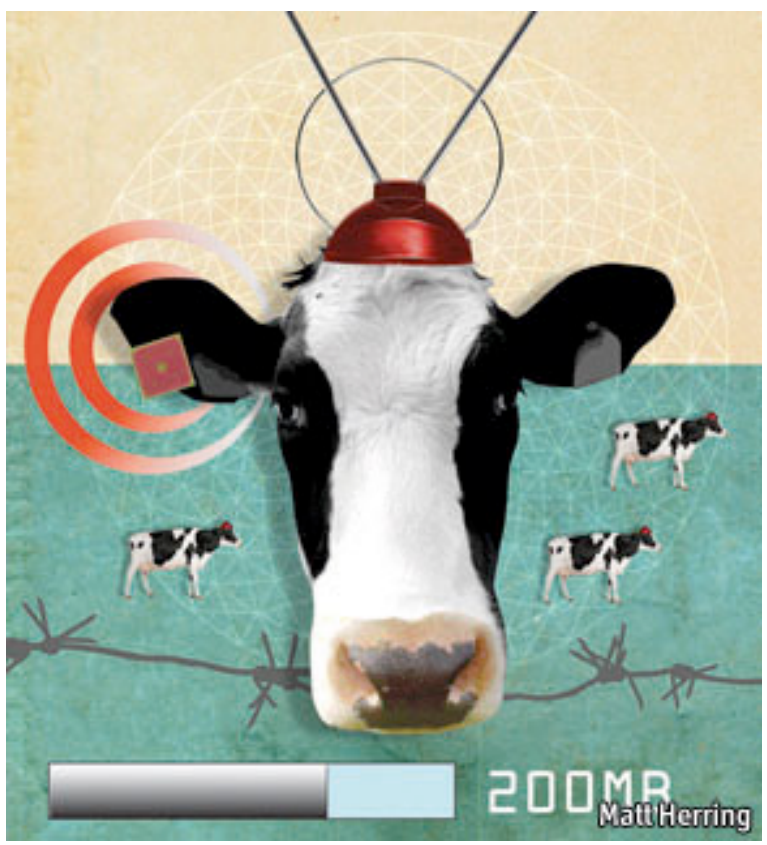

Source: The Economist, 2010. 


\section{Mumbai: A Tale of Two Cities}

While greater efficiencies and new business models will have a positive economic impact, the human aspect, in many ways, will provide the most important benefit of loT. One of the areas where loT can make a significant difference is in closing the poverty gap. Dr. C.K. Prahalad's book, The Fortune at the Bottom of the Pyramid: Eradicating Poverty Through Profits, provides some mind-boggling statics comparing Dharavi (the poorest neighborhood in Mumbai) to Warden Road (the better side of the city just blocks away).

The amount people from Dharavi pay for municipal-grade water is $\$ 1.12$ per cubic meter. This compares to $\$ 0.03$ for residents of Warden Road. The injustice is clear: the poor people of Mumbai pay 37 times more for water (a basic human necessity). ${ }^{15}$

The main source of the disparity is the higher cost of delivering utility services to poorer neighborhoods because of infrastructure inefficiencies, problems such as leaks, and theft. According to an article in The Wall Street Journal, "Seven years ago, more than 50 percent of the power distributed by North Delhi Power Ltd. wasn't paid for by customers. A key challenge for power companies is reducing theft by India's poor."

Figure 5. Electric Utility Inefficiencies in India.

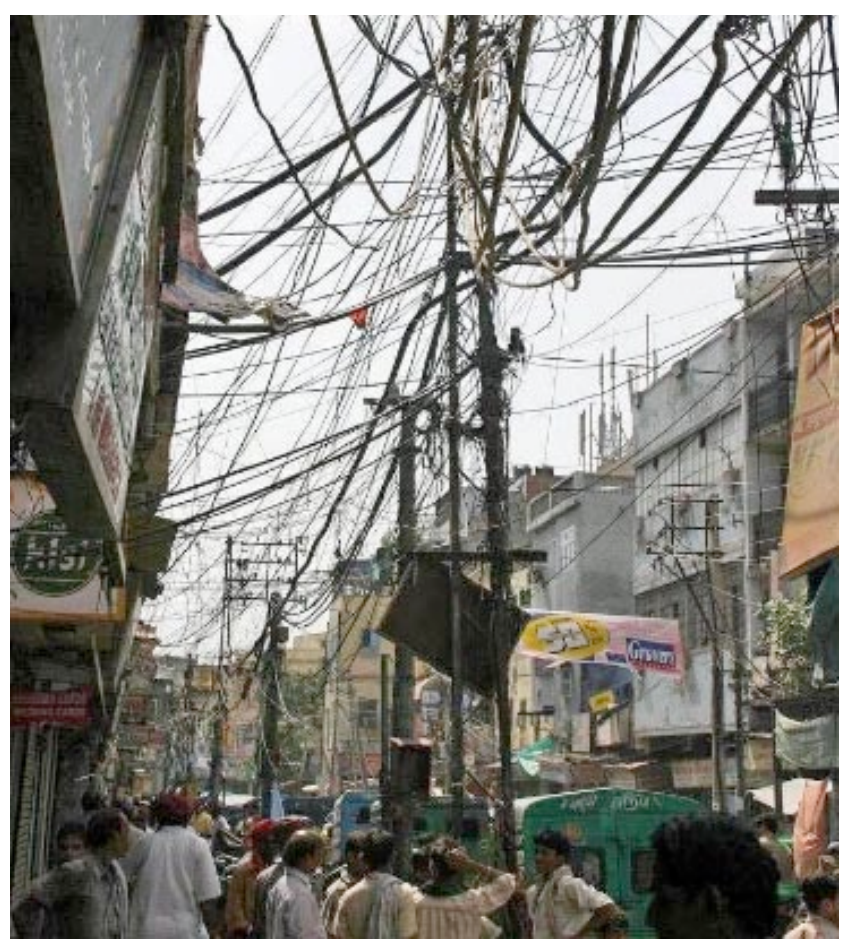

Source: The Wall Street Journal, 2009.

IoT, because of its ubiquitous sensors and connected systems, will provide authorities with more information and control in order to identify and fix these problems. This will allow utilities to operate more profitably, giving them extra incentive to improve infrastructures in poorer neighborhoods. More efficiency will also allow for lower prices, which, in turn, will encourage those taking services for free to become paying customers. ${ }^{16}$ 


\section{Better Quality of Life for the Elderly}

The world's population is aging. In fact, approximately 1 billion people age 65 and older will be classified as having reached "non-working age" by the middle of the century. ${ }^{17}$ loT can significantly improve quality of life for the surging number of elderly people. For example, imagine a small, wearable device that can detect a person's vital signs and send an alert to a healthcare professional when a certain threshold has been reached, or sense when a person has fallen down and can't get up.

\section{Challenges and Barriers to loT}

Several barriers, however, have the potential to slow the development of loT. The three largest are the deployment of IPv6, power for sensors, and agreement on standards.

Deployment of IPv6. The world ran out of IPv4 addresses in February 2010. While no real impact has been seen by the general public, this situation has the potential to slow loT's progress since the potentially billions of new sensors will require unique IP addresses. In addition, IPv6 makes the management of networks easier due to auto configuration capabilities and offers improved security features.

Sensor energy. For loT to reach its full potential, sensors will need to be self-sustaining. Imagine changing batteries in billions of devices deployed across the planet and even into space. Obviously, this isn't possible. What's needed is a way for sensors to generate electricity from environmental elements such as vibrations, light, and airflow. ${ }^{18}$ In a significant breakthrough, scientists announced a commercially viable nanogenerator - a flexible chip that uses body movements such as the pinch of a finger to generate electricity-at the 241 st National Meeting \& Exposition of the American Chemical Society in March $2011 .^{19}$

"This development [the nanogenerator] represents a milestone toward producing portable electronics that can be powered by body movements without the use of batteries or electrical outlets. Our nanogenerators are poised to change lives in the future. Their potential is only limited by one's imagination."

\section{Zhong Lin Wang}

Lead Scientist, Georgia Institute of Technology

Standards. While much progress has been made in the area of standards, more is needed, especially in the areas of security, privacy, architecture, and communications. IEEE is just one of the organizations working to solve these challenges by ensuring that IPv6 packets can be routed across different network types.

It is important to note that while barriers and challenges exist, they are not insurmountable. Given the benefits of loT, these issues will get worked out. It is only a matter of time.

\section{Next Steps}

As often happens, history is repeating itself. Just as in the early days when Cisco's tagline was "The Science of Networking Networks," loT is at a stage where disparate networks and a multitude of sensors must come together and interoperate under a common set of standards. 
This effort will require businesses, governments, standards organizations, and academia to work together toward a common goal.

Next, for loT to gain acceptance among the general populace, service providers and others must deliver applications that bring tangible value to peoples' lives. loT must not represent the advancement of technology for technology's sake; the industry needs to demonstrate value in human terms.

In conclusion, loT represents the next evolution of the Internet. Given that humans advance and evolve by turning data into information, knowledge, and wisdom, loT has the potential to change the world as we know it today - for the better. How quickly we get there is up to us.

For more information, please contact Dave Evans, Cisco's chief futurist and chief technologist for Cisco IBSG, at devans@cisco.com.

The following individuals made critical contributions to the development of this paper:

- Scott Puopolo, vice president, Cisco IBSG Service Provider Practice

- Jawahar Sivasankaran, senior manager, Cisco IT Customer Strategy \& Success group

- JP Vasseur, distinguished engineer, Cisco Emerging Technologies

- Michael Adams, Cisco IBSG Communications Strategy Practice

\section{Endnotes}

1. Source: Wikipedia, 2011.

2. Source: Cisco IBSG, 2011.

3. Sources: U.S. Census Bureau, 2010; Forrester Research, 2003.

4. Source: Wikipedia, 2010.

5. Sources: Cisco IBSG, 2010; U.S. Census Bureau, 2010.

6. While no one can predict the exact number of devices connected to the Internet at any given time, the methodology of applying a constant (Internet doubling in size every 5.32 years) to a generally agreed-upon number of connected devices at a point in time (500 million in 2003) provides an estimate that is appropriate for the purposes of this paper. Sources: "Internet Growth Follows Moore's Law Too," Lisa Zyga, PhysOrg.com, January 14, 2009, http://www.physorg.com/news151162452.html; George Colony, Forrester Research founder and chief executive officer, March 10, 2003, http://www.infoworld.com/t/platforms/forrester-ceo-web-services-next-it-storm-873

7. Source: "Planetary Skin: A Global Platform for a New Era of Collaboration," Juan Carlos Castilla-Rubio and Simon Willis, Cisco IBSG, March 2009, http://www.cisco.com/web/about/ac79/docs/pov/Planetary Skin POV vFINAL spw je_2.pdf

8. Source: World Internet Stats: Usage and Population Statistics, June 30, 2010.

9. Sources: Cisco, 2010; HP, 2010. 
10. Source: “The Networked Pill," Michael Chorost, MIT Technology Review, March 20, 2008, http://www.technologyreview.com/biomedicine/20434/?a=f

11. Source: "Researchers Debut One-Cubic-Millimeter Computer, Want to Stick It in Your Eye," Christopher Trout, Endadget, February 26, 2011, http://www.engadget.com/2011/02/26/researchers-debut-one-cubic-millimetercomputer-want-to-stick-i/

12. Cisco's Internet Routing in Space (IRIS) program uses the Cisco Space Router to extend IP access using satellites. The router eliminates the need to send data to and from an extra ground station, which can be expensive and time-consuming. Further, Cisco Space Routers extend IP access to areas not covered by traditional ground networks or 3G networks, delivering consistent and pervasive IP capabilities regardless of geographic location.

13. Source: "The Discovery of the Molecular Structure of DNA," NobelPrize.org.

14. Source: "Augmented Business," The Economist, November 2010.

15. Source: Fortune at the Bottom of the Pyramid: Eradicating Poverty Through Profits, Dr. C.K. Prahalad.

16. Source: "India Has Its Own Kind of Power Struggle," The Wall Street Journal, Jackie Range, August 7, 2009.

17. Source: United Nations, 2010.

18. Source: "Smart Dust Sensor Network with Piezoelectric Energy Harvesting," Yee Win Shwe and Yung C. Liang, ICITA, 2009, http://www.icita.org/papers/34-sg-Liang217.pdf

19. Source: "First Practical Nanogenerator Produces Electricity with Pinch of the Fingers," PhysOrg.com, March 29, 2011, http://www.physorg.com/news/2011-03nanogenerator-electricity-fingers.html

\section{More Information}

Cisco Internet Business Solutions Group (IBSG), the company's global consultancy, helps CXOs from the world's largest public and private organizations solve critical business challenges. By connecting strategy, process, and technology, Cisco IBSG industry experts enable customers to turn visionary ideas into value.

For further information about IBSG, visit http://www.cisco.com/go/ibsg.

\section{$, 1 \mid 1,1 / 1$, CISCO.}

Americas Headquarters

Cisco Systems, Inc.

San Jose, CA
Asia Pacific Headquarters

Cisco Systems (USA) Pte. Ltd

Singapore
Europe Headquarters

Cisco Systems International BV Amsterdam,

The Netherlands

Cisco has more than 200 offices worldwide. Addresses, phone numbers, and fax numbers are listed on the Cisco Website at www.cisco.com/go/offices.

Cisco and the Cisco Logo are trademarks of Cisco Systems, Inc. and/or its affiliates in the U.S. and other countries. A listing of Cisco's trademarks can be found at www.cisco.com/go/trademarks. Third party trademarks mentioned are the property of their respective owners. The use of the word partner does not imply a partnership relationship between Cisco and any other company. (1005R) 